\title{
A REAL-TIME SCHEDULING POLICY FOR MULTIMEDIA APPLICATIONS ON BROADCAST NETWORKS
}

\author{
Jau-Hsiung Huang and Ing-Chau Chang \\ Communications and Multimedia Lab. \\ Department of Computer Science and Information Engineering \\ National Taiwan University, Taipei, Taiwan
}

\begin{abstract}
Multimedia applications have become some of the most important applications on high speed local and metropolitan area networks. One of the major characteristics of multimedia traffic is the real-time constraint of packets. Furthermore, in multimedia applications, packets of different applications may have different levels of priority. Hence, a proper scheduling policy is necessary to schedule packets with different priorities and real-time constraints. This paper presented a scheduling policy for real-time traffic with priorities on broadcast networks to reduce the probability of packet loss due to exceeding the real-time constraint. Moreover, if packet loss is unavoidable, this scheduling policy will avoid dropping the packets with high priorities.
\end{abstract}

\section{Introduction}

The most significant characteristic of multimedia applications is probably the real-time constraint. That is, a packet generated at the source should be received by the receiver before a specified time which is denoted as the deadline. Otherwise, this packet would become useless and will be dropped. Besides the real-time constraint, different applications may have different degrees of importance and hence will be assigned different levels of priority. Since it is impossible for any scheduling policy to avoid dropping packets for multimedia applications when the network load is heavy; hence, the good scheduler should drop packets with lower priorities first if packet dropping is unavoidable. We define normalized penalty, denoted as NP, as

$$
N P=\frac{\text { sum of priorities of all lost packets }}{\text { sum of priorities of all packets }}
$$

Clearly, a good scheduling policy should be able to keep the value of NP as small as possible.

There have been works [1-5] on the design of scheduling algorithms for applications with real-time constraint. However, these works treat all packets with equal importance; hence, their scheduling policies depend only on the real-time constraints. The scheduling policy proposed in this paper will take into account both the real-time constraint and the priority values of packets.

\section{System Model and Scheduling Policy}

In this work, the network architecture considered is a broadcast network such as token ring or token bus such that stations will transmit in turn. Since whether a packet should be sent urgently or not depends on the deadline and the current time; hence, we define urgentness of a packet to be the difference between its deadline and the current time.

In order to design the scheduling policy, each packet will be assigned a cost which is a function of the priority and the urgentness of the packet. Hence, a packet with a larger cost should be transmitted earlier. All packets in the local queue of each station will maintain a sorted sequence based on the costs of packets. Then, the scheduling policy will find the packet with the largest cost in the entire network to be transmitted first. Note that clock synchronization among stations is not necessary since the urgentness does not refer to the absolute time; instead, it refers only to the time difference. We define the following notation:

$t$ : the current time.

$\mathrm{P}_{\mathrm{i}}$ : the priority value of the packet in the ith position of the local queue. 
$\mathrm{T}_{\mathrm{i}}$ : the deadline of the packet in the ith position of the local queue.

$\mathrm{C}_{\mathrm{i}}(\mathrm{t})$ : the cost of the packet in the ith position of the local queue at time $\mathrm{t}$.

$f\left(P_{i}, U_{i}\right)$ : the cost function of a packet with priority $P_{i}$ and urgentness $U_{i}\left(C_{i}(t)=f\left(P_{i}, T_{i}-t\right)\right)$.

Note that since the urgentness of a packet decreases over time, hence the cost of a packet should increase over time. Based on this observation, a good cost function should possess the following two criteria.

\section{Criteria:}

(1) The cost function should increase monotonically when the urgentness decreases.

(2) For two packets with priorities $P_{i}$ and $P_{j}$ and deadline $T_{i}$ and $T_{j}$, respectively; if $f\left(P_{i}, T_{i}-t\right)>f\left(P_{j}, T_{j}-t\right)$ at time $t$, then $f\left(P_{i}, T_{i}-t-\tau\right)>f\left(P_{j}, T_{j}-t-\tau\right)$ for all $\tau$ as long as both $\mathrm{T}_{\mathrm{i}}-\mathrm{t}-\tau>0$ and $\mathrm{T}_{\mathrm{j}}-\mathrm{t}-\tau>0$.

The physical interpretation of the second criterion is that if packet $i$ is queued ahead of packet $j$ at time $t$; then, after $\tau$ units of time, the queueing order based on the costs of these two packets will remain unchanged even though the costs of both packets have been changed. There are many reasonable cost functions which satisfy the first criterion. For example, $f\left(P_{i}, T_{i}\right)=\frac{P_{i}}{T_{i}-t}$ is a good example which states that the cost function is proportional to the priority and inverse proportional to the urgentness. However, such a cost function does not satisfy the second criterion.

The problem of a cost function violating the second criterion is that the packet sequence in the queue will change over time. That is, even though packet $i$ is ahead of packet $j$ at time $t$, this queueing order may be changed $\tau$ units of time later. Hence, the scheduler will have to recompute the costs of all packets in the queue all the time to find the proper queueing order for that moment of time. This will incur a significant processing overhead to make it infeasible. Based on this observation, we choose the following exponential function as the cost function:

$$
\begin{aligned}
C_{i} & =f\left(P_{i}, U_{i}\right)=\left(P_{i}\right)^{r} * \exp \left(-\frac{U_{i}}{k}\right) \\
& =\left(P_{i}\right)^{r} * \exp \left(-\frac{T_{i}-t}{k}\right)
\end{aligned}
$$

where $r$ and $k$ are two parameters. It can easily be shown that this cost function satisfies the criteria stated above.

In the following, we will compare this cost function, under various values of $r$ and $k$, with EDF(Earliest Deadline First) [3] and LPF (Largest Priority First) to assess their performance. For the rest of the paper, we will refer LCF (Largest Cost First) as the scheduling policy using Eq.(1) as the cost function. In fact, both EDF and LPF are variants of LCF. For instance, by setting $r=0$ in Eq.(1) gives the equivalent effect of $E D F$ and by setting $k=\infty$ gives the equivalent effect of LPF.

\section{Performance Comparisons Among Policies}

In this section, several simulations will be run to compare the performance of various policies under the following conditions.

1. Poisson arrival process with infinite buffer.

2. 10 stations in the network.

3. Fixed packet transmission time (unity) and the propagation delay between stations equals 0.001 .

4. The real-time constraint is uniformly distributed over $(0$, 10] with mean equals 5 time units.

5. The priority assignment is from the set of $\{1,5,10\}$ with equal probability.

As shown in Fig.1, we see that EDF does not achieve the smallest NP while LCF with $k=3$ outperforms other policies for most of the loads. Figs. 2 and 3 show the packet loss probability using LCF policy for $k=1$ and 3 , respectively. From these figures, it is shown that a larger $k$ enlarges the difference of the packet loss probability between high and low priority packets and provides a smaller packet loss probability to high priority packets at the cost of low priority packets. Similarly, Fig. 4 shows the packet loss probability using LCF policy for $\mathrm{k}=3$ and $\mathrm{r}=2$. It is shown in this figure that a larger $r$ also enlarges the difference of the packet loss probability between high and low priority packets. Moreover, as shown in Fig.4, the packet loss probability of the high priority packets is kept very small even when the network is very heavily loaded. This provides a very good performance to high priority packets. From these studies, the following two conclusions are drawn: 
(1) If packet priority is more important than the urgentness, then a larger $r$ or a larger $k$ should be chosen.

(2) A larger value of $r$ or $k$ will broaden the performance gap, with respect to the packet loss probability, between packets with different priorities.

\section{References}

[1] Jon M. Pehá and Fouad A. Tobagi, "A Cost_Based Scheduling Algorithm to Support Integrated Services," Proc. IEEE INFOCOM'91, pp.741-753, 1991.

[2] Partha P. Bhattacharya and Anthomy Ephremides, "Optimal Scheduling with Strict Deadlines," IEEE Trans. on Automatic Control, Vol 34, No 7, pp. 721-728, July 1989.

[3] Shivenra S. Panwar, Don Towsley, and Jack K. Wolf, "Optimal Scheduling Policies for a Class of Queues with Customer Deadlines to the Beginning of Service," Journal of the ACM, Vol 35, No 4, pp.832-844, Oct. 1988.

[4] L. Yao, W. Zhao, "Performance of an Extended IEEE 802.5 Protocol in Hard Real-Time Systems," Proc. IEEE INFOCOM'91, pp. 469-478, 1991

[5] A.K. Mok and M. L. Dertouzos. "Multiprocessor scheduling in a hard real-time environment.", Proc. the Seventh Texas Conference on Computing Systems, November 1978 .

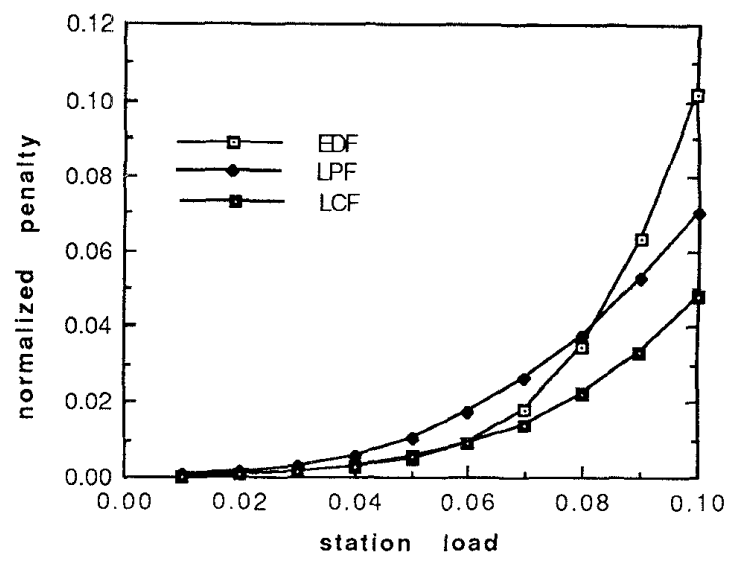

Fig.1. Normalized penalty for various scheduling policies.

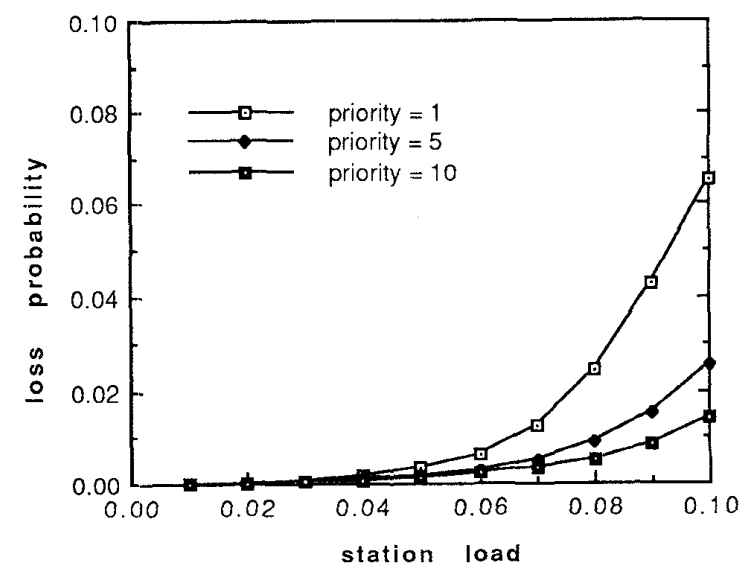

Fig. 2. Packet loss probability for various priorities when $k=1$ and $r=1$. 


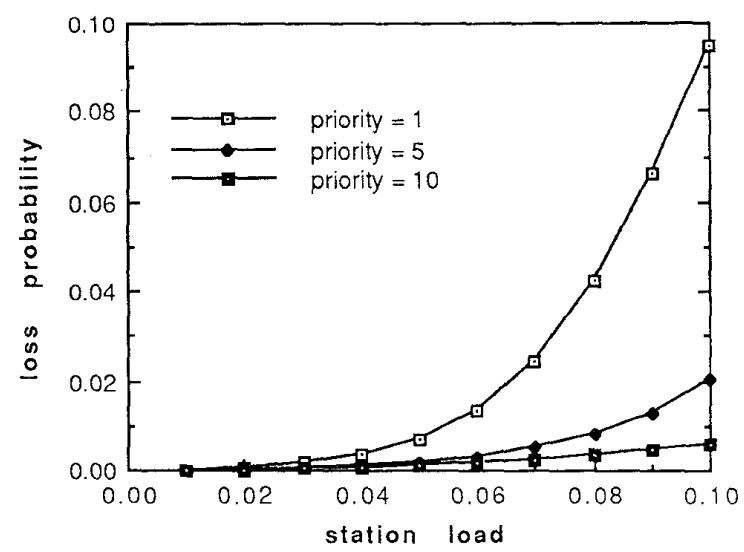

Fig. 3. Packet loss probability for various priorities when $\mathrm{k}=3$ and $\mathrm{r}=1$.

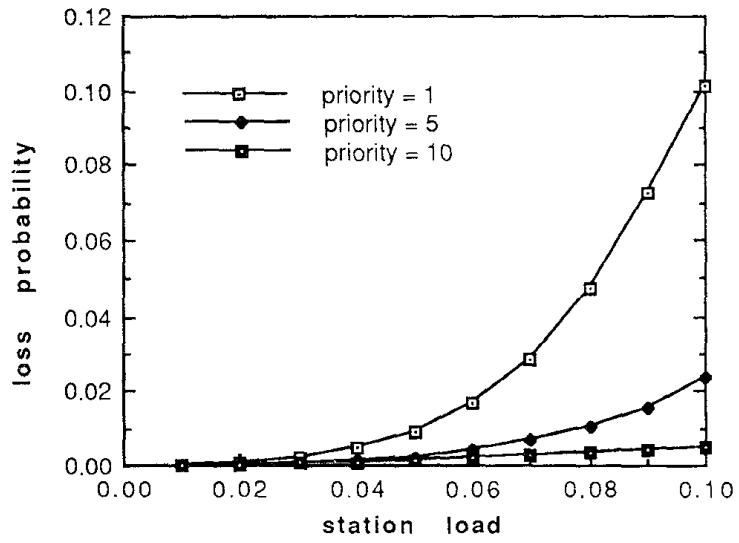

Fig. 4. Packet loss probability for various priorities when $\mathrm{k}=3$ and $\mathrm{r}=2$. 